\title{
To inhibit growth and migration of glioma cells through down regulation glucose metabolism related to aquaporin (AQP)-1
}

\author{
Xiangming Liu ${ }^{1}$, Jinglong Chen ${ }^{2}$, Jinqian Zhang ${ }^{3}$ \\ ${ }^{1}$ Department of Neurosurgery, Beijing Tiantan Hospital, Capital Medical University, Beijing 100050, China; ${ }^{2}$ Department of Oncology, Beijing \\ Ditan Hospital, Capital Medical University, Beijing 100015, China; ${ }^{3}$ Department of Medical Experiment Center, Guangdong Second Provincial \\ General Hospital, Guangzhou 510317, China \\ Contributions: (I) Conception and design: J Zhang; (II) Administrative support: J Chen; (III) Provision of study materials or patients: X Liu; (IV) \\ Collection and assembly of data: X Liu, J Chen; (V) Data analysis and interpretation: J Chen, J Zhang; (VI) Manuscript writing: All authors; (VII) \\ Final approval of manuscript: All authors. \\ Correspondence to: Prof. Jinqian Zhang. Department of Medical Experiment Center, Guangdong Second Provincial General Hospital, No. 466 \\ Xingang Middle Road, Haizhu District, Guangzhou 510317, China. Email: jingwanghou@163.com; Dr. Jinglong Chen. Department of Oncology, \\ Beijing Ditan Hospital, Capital Medical University, No. 8 Jingshun East Street, Chaoyang District, Beijing 100015, China. Email: dtzlzx@sina.com.
}

\begin{abstract}
Background: Glioma is one of highly aggressive and fastest growing human tumors. aquaporin (AQP)-1 is a critical factor in cell function, especially in glioma cells. However, its mechanism is unknown. Here, we explored growth and migration mechanism of glioma cells associated with glucose metabolism regulated by AQP-1.

Methods: The growth and migration of glioma cells were analyzed after up or down regulation of AQP-1 expression. The expression of glucose metabolism-related proteins G6PC (glucose-6-phosphatase, catalytic subunit), GK (glycerol kinase), and GOT1 (glutamic-oxaloacetic transaminase 1) were examined. Glucose production of glioma cells was determined with colorimetric glucose oxidase method.

Results: Our work indicated that AQP-1 facilitated growth and migration of glioma cells by regulating glucose metabolism, which was related to down-expression of gluconeogenesis gene G6PC, GK and GOT1. Interference on AQP-1 expression suppressed these processes of glioma cells.

Conclusions: Therefore, our data demonstrated that down regulation of AQP-1 can induce the inhibition of glioma cell growth and migration through the pathway related to gluconeogenesis gene G6PC, GK and GOT1, which may promote the glucose metabolism. AQP-1 is the potential novel target for treatment of glioma.
\end{abstract}

Keywords: Growth; migration; glioma; aquaporin (AQP); glucose metabolism

Submitted Mar 03, 2018. Accepted for publication Jun 15, 2018.

doi: $10.21037 /$ tcr.2018.11.02

View this article at: http://dx.doi.org/10.21037/tcr.2018.11.02

\section{Introduction}

Glioma come from spine or brain, and is the most common tumor in brain (1). Gliomas include approximately $80 \%$ of malignant tumors in brain and $30 \%$ in central nervous system (CNS) (2). It is related to poor prognosis, especially in older patients with high-grade tumors. The one-year survival rate for patients with glioma is about $30 \%$, and the two-year survival rate is around 14\% (3). Young patients with high-grade gliomas tend to have a better prognosis, if complete resection of tumor is performed (4). In patients with anaplastic astrocytoma, the median survival is around 2-3 years, but in glioblastoma multiformes (GBMs) may be only approximately one year (4).

It is a challenge to identify the potential targets for treatment on GBM. The mechanistic insight associated establishment and progression of tumor was provided according to novel over-expressed or mutated molecules, which might be the new anticancer aims to drugs research (5).

Aquaporins (AQPs) are a proteins family, and key function of them is water channels and channel-forming (6). 
Moreover, ten AQPs have been found today (7). They have been distinguished into two subgroups according to functional studies: AQPs are selective water channels, not only the aquaglyceroporins are transport glycerol but also water (8). AQP-1 is owned by the AQP family of water channel and an aquaglyceroporin, is a widely expressed water channel, whose is permeable to water as well as to none charged solutes for example lactate. The expression of AQP-1 in GBMs increased compared with the normal tissues of brain. AQPs play a vital role in shuttling of $\mathrm{H}_{2} \mathrm{O}$ and $\mathrm{H}^{+}$between the space from extracellular to intracellular, cell motility, and edema (9). The common feature of cancer cells is accumulation of lactate and increased glucose uptake, even in normoxic conditions such as aerobic glycolysis or the Warburg effect (10). In cancer cells, lactate may be as a source of $\mathrm{NADH}$ and energy substrate or scavenger of reactive oxygen species (ROS). In tumors, glutaminolytic and glycolytic cells could produce and export lactate, which may be as an oxidative fuel recycled by tumors cells in oxidative status (11).

The metastatic cascade process is the central of cell migration, which need coordinate with the behavior of transporters, signaling cascades, cytoskeletal elements and ion channels. Aquaporins and ion transport play the key role of in invasion and migration of tumor cell, by causing changes of volume through regulating signaling associated with $\mathrm{H}^{+}$and $\mathrm{Ca}^{2+}$. In carcinoma, not only ion transports overexpression are common, but also activated. Therefore, cell migration may be the potential target for treatment of glioma, and ion transports are attractive candidate aims (12). Oedema formation is a chief donor to the morbidity and mortality related to malignant brain tumors, or liver, spleen and testicle (13). The high glycerol concentration induces viscosity, which could reduce the speed of biochemical processes in tumor cells (14). Recent studies demonstrate that aquaporins might be a capable target for treatment on cancer $(15,16)$.

There are four chief reactions involved in gluconeogenesis, including G6Pase (glucose-6-phosphatase), F1, 6BPase (fructose-1, 6-bisphosphatase), PEPCK (phosphoenolpyruvate carboxykinase), and PC (pyruvate carboxylase). The regulation of these enzymes in transcriptional level is vital to effectively induce gluconeogenesis. Then the key rate-limiting enzyme of these four reactions were investigated in this work, such as G6PC (glucose-6-phosphatase, catalytic subunit), GK (glycerol kinase), and GOT1 (glutamic-oxaloacetic transaminase 1).

Therefore, we investigated that the effect of AQP-1 expression on human glioma cell lines. The propose of our work was to study the AQP-1 expression in glioma cells, and to ascertain the potential roles of AQP-1 associated with glucose metabolism on glioma progression and to evaluate the possible options for the treatment on glioma related to AQP-1.

\section{Methods}

\section{Cell culture}

Both cell lines of human glioma U87 and U251 were obtained from ATCC (American Type Culture Collection) and cultured with DMEM medium blending FBS (Gibco, Carlsbad, CA, USA), streptomycin, and penicillin in incubator with $5 \% \mathrm{CO}_{2}$ humidified atmosphere at $37{ }^{\circ} \mathrm{C}$ followed the methods of Liu et al. 2017 (17).

\section{Plasmid constructs and DNA purification, transfection}

The plasmids of overexpression of AQP-1 were established by standard method (18). The pCMV-4 plasmids were obtained from Clontech. A total of $4 \times 10^{5}$ of cultured cells in each well of 6 -well plates was added to $4 \mu \mathrm{g}$ plasmids using Lipofectamine 3000.

One day before transfection, seed $0.5-2 \times 10^{5}$ cells per well in $500 \mu \mathrm{L}$ of growth medium without antibiotics to attain $90-95 \%$ confluence at the time of transfection. For each transfection sample, prepare DNA-Lipofectamine 3000 complexes as follows: dilute $0.8 \mu \mathrm{g}$ DNA in $50 \mu \mathrm{L}$ Opti-MEM I (Invitrogen Catalog No. 31985, USA) without serum, and then mix gently. Mix the stock Lipofectamine 3000 gently before use, and then dilute $2 \mu \mathrm{L}$ in $50 \mu \mathrm{L}$ OptiMEM I. Mix gently and let it stand for $5 \mathrm{~min}$ at room temperature. Five minutes after dilution of Lipofectamine 3000 , combine the diluted DNA with the diluted lipid (total volume is $100 \mu \mathrm{L}$ ). Mix gently and let it stand for $20 \mathrm{~min}$ at room temperature to allow the DNA-Lipofectamine 3000 lipoplexes to form. The solution may appear cloudy, but this will not inhibit transfection. DNA-Lipofectamine 3000 complexes are stable for $6 \mathrm{~h}$ at room temperature. Add $100 \mu \mathrm{L}$ of transfection complex to each well containing cells and medium. Mix gently by rocking the plate back and forth. Incubate the cells at $37{ }^{\circ} \mathrm{C}$ in a humidified incubator with $5 \% \mathrm{CO}_{2}$ for $48 \mathrm{~h}$ until they are ready to assay.

\section{siRNA of $A Q P-1$}

The synthetic siRNAs were obtained from Santa Cruz Inc. (Santa Cruz, CA, USA), and the most effective siRNA of AQP-1 (sc-29711) was used in vitro. The siRNA of negative control sc- 37007 was selected. A total of $4 \times 10^{5}$ of cultured cells in each well of 6 -well plates was added to $4 \mu \mathrm{g}$ siRNA using Lipofectamine 3000. 


\section{Western blot analysis}

The proteins were extracted from lysed cells, and then were detected with sodium dodecyl sulfate-polyacrylamide (SDSPAGE) gel. After that, the proteins were transferred to $0.45 \mu \mathrm{m}$ polyvinylidene fluoride (PVDF) membrane, then it was blocked with skim milk solution (5\% in TBST) and incubated using primary antibody: anti-AQP-1, G6PC, GK and GOT1 (1:3,000 dilutions, Cell Signaling, Danvers, MA, USA), $\beta$-actin (1:4,000 dilutions, Santa Cruz Biotechnology, Santa Cruz, CA, USA). Furthermore, the membranes were at probed with proper secondary antibodies (1:6,000 dilutions, Santa Cruz Biotechnology) (17).

\section{Detection of glucose production}

Glucose production of cells was detected with colorimetric glucose oxidase method. The isolated cells were cultured with medium maintained gluconeogenesis (19). Glucose production of cells was measured by the glucose oxidase colorimetric method.

A certain amount of 1-thio- $\beta$-d-glucose were incubated with different amounts of GOx in citrate buffer $(10 \mathrm{mM}$, $\mathrm{pH} 4.0$ ) for $1 \mathrm{~h}$ at $37{ }^{\circ} \mathrm{C}$. After that, $20 \mu \mathrm{L} \mathrm{Cd}{ }^{2+} / \mathrm{Na}_{3} \mathrm{PO}_{4}$ mixture was added to the samples. Subsequently, the mixed solution was added with $20 \mu \mathrm{L}$ of $5 \mathrm{mM}$ TMB and then diluted to $200 \mu \mathrm{L}$ by acetate buffer ( $200 \mathrm{mM}, \mathrm{pH} 4.0)$ and illuminated under visible light irradiation $(\lambda \geq 400 \mathrm{~nm})$ for $10 \mathrm{~min}$ to allow development of the blue color, and the absorbance of the oxidized TMB (oxTMB) at $652 \mathrm{~nm}$ was measured.

\section{Analysis of proliferation and clonogenicity}

The MTS assay was used to analyze the ability of cell growth. Moreover, the clonogenicity assay was also performed. The colonies of glioma cells were fixed with formalin, and then stained with crystal violet (17).

The proliferation ability of glioma cells was assessed using MTS kit. Cells were exposed to vehicle control (DMSO) or to $4 \beta \mathrm{HWE}$ at concentrations of $1,2,5$ and $10 \mu \mathrm{g} / \mathrm{mL}$ for $24 \mathrm{~h}$. The cells were then exposed to MTS solution (CellTiter 96 Aqueous One Solution, Promega, Madison, WI, USA) and allowed to incubate for $2 \mathrm{~h}$ at $37^{\circ} \mathrm{C}$. The product was measured at $490 \mathrm{~nm}$ absorbance using a Dynex MRX Model 96 Well Plate Reader (MTX Lab Systems, Inc., Vienna, VA, USA).

The colonies of cells were fixed firstly, and then determined with crystal violet stain. Then the sums of colonies were counted. Over- or down-expression of AQP-1 were induced for assessing its molecular biological functions in glioma cells with several experiments. The clonogenicity experiment was performed, cells colonies were fixed using formalin and then stained by crystal violet (Sigma, USA). Furthermore, the number of obvious colonies were figured out.

\section{Determine of migration in glioma cells}

The migration ability of glioma cells was measured with wound scratch experiment. At first, glioma cells were seed into 24-well tissue culture plate at $5 \times 10^{3}$ cells per well that after $24 \mathrm{~h}$ of growth, they should reach $70-80 \%$ confluence as a monolayer. Then, gently and slowly scratch the monolayer with a new $1 \mathrm{~mL}$ pipette tip across the center of the well. After scratching, gently wash the well twice with medium to remove the detached cells. Furthermore, glioma cells were cultured with fresh medium for additional $48 \mathrm{~h}$. After washing the cells twice with $1 \times$ PBS, the cells were fixed with $3.7 \%$ paraformaldehyde for $30 \mathrm{~min}$, then stained with $1 \%$ crystal violet in $2 \%$ ethanol for $30 \mathrm{~min}$. Take photos for the stained monolayer were performed on a microscope. The gap distance can be quantitatively evaluated, the migration glioma cells were counted (17).

\section{Statistics}

Data were displayed as mean $\pm \mathrm{SD}$. The significance differences between data were estimated with two-tailed Student's $t$-test or one-way ANOVA (analysis of variance). For comparison of quantitative data, $t$-tests or ANOVA were conducted between groups. Medians were compared between groups through Kruskal-Wallis ANOVA. SPSS software (version 18.0; SPSS Inc., Chicago, IL, USA) was used to perform all analyses. $\mathrm{P}<0.05$ were considered significant.

\section{Results}

\section{$A Q P-1, G 6 P C, G K$ and GOT1 proteins expression in glioma cells}

The expression level of G6PC, GK, GOT1, and AQP-1 proteins in glioma cells increased significantly induced by overexpression of AQP-1, and was much more than control group. At siRNA AQP-1 group, the expression level of G6PC, GK, GOT1, and AQP-1 proteins decreased obviously compared with the control group (Figure 1). It indicated the expression level of G6PC, GK, GOT1, and AQP-1proteins could be induced by the treatment with overexpression of AQP-1 in glioma cells, but reduced by siRNA of AQP-1. 

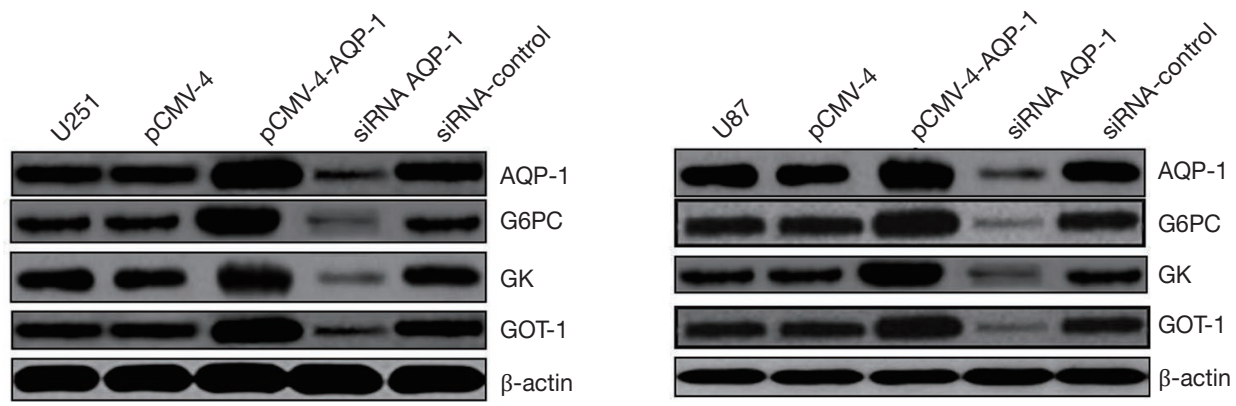

Figure 1 The protein expression of AQP-1, G6PC, GK and GOT1 in glioma cells. The expression level of G6PC, GK, GOT1, and AQP-1 proteins in glioma cells increased significantly induced by overexpression of AQP-1, and was much more than control group. At siRNA AQP-1 group, the expression level of G6PC, GK, GOT1, and AQP-1 proteins decreased obviously compared with the control group. AQP, aquaporin; G6PC, glucose-6-phosphatase, catalytic subunit; GK, glycerol kinase; GOT1, glutamic-oxaloacetic transaminase 1.
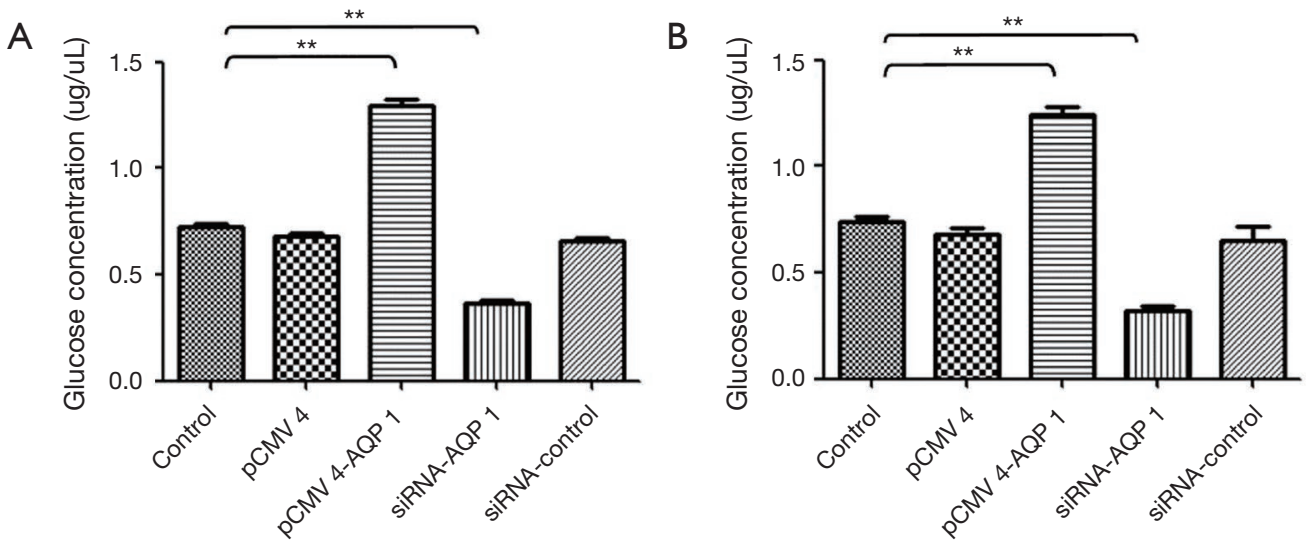

Figure 2 AQP-1 enhances glucose production in glioma cells. Compared with control, the amounts of glucose production in glioma cell lines U251 (A) and U87 (B) treat with overexpression of AQP-1 enhanced obviously $(\mathrm{P}<0.01)$. Moreover, at siRNA AQP-1 group, the amounts of glucose production decreased obviously. The data are presented as means \pm SD from three independent experiments. ${ }^{* *}, \mathrm{P}<0.01$; $\mathrm{P}$ value was generated using Kruskal-Wallis ANOVA. AQP, aquaporin; ANOVA, analysis of variance.

\section{AQP-1 enhances glucose production in glioma cells}

Compared with control, the amounts of glucose production in glioma cell lines U251 (Figure 2A) and U87 (Figure 2B) treat with overexpression of AQP-1 enhanced obviously $(\mathrm{P}<0.01)$. Moreover, at siRNA AQP-1 group, the amounts of glucose production decreased obviously (Figure 2). These results showed that the amounts of glucose production could be induced by the treatment with overexpression of AQP-1 in glioma cells, but reduced by siRNA of AQP-1.

\section{AQP-1 promoted glioma cell growth}

Glioma cells $\left(3 \times 10^{3}\right)$ were added into 96 -well plates and cultured for $24 \mathrm{~h}$, then transfection with pCMV-4, pCMV-
4-AQP-1, and the siRNA of AQP-1 or siRNA control. We conduct the MTS assay to assess the effect of over expression or siRNA AQP-1 on proliferation of glioma cells. Compared with miR-NC cells, proliferation of U251 (Figure 3A) and U87 (Figure 3B) cells would be accentuated when they were transfected with pCMV-4-AQP-1, respectively. Moreover, the siRNA of AQP-1 attenuated these processes of glioma cells (Figure 3).

Furthermore, the colony formation assay revealed that AQP-1 exhibited significantly facilitated ability for colony formation compared with the control group in both cell lines U251 (Figure 4A,B) and U87 (Figure 4B,C) $(\mathrm{P}<0.001$ ). But, down-regulation of AQP-1 decreased significantly ability of colony formation in both cell lines. These results suggested that AQP-1 also suppressed the clonogenicity 
A

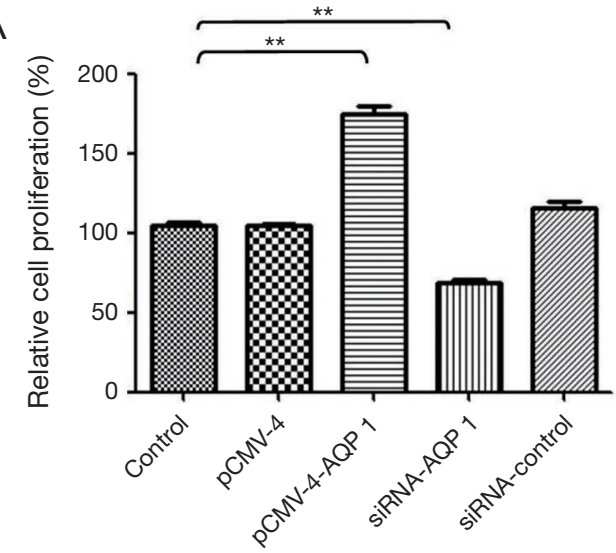

B

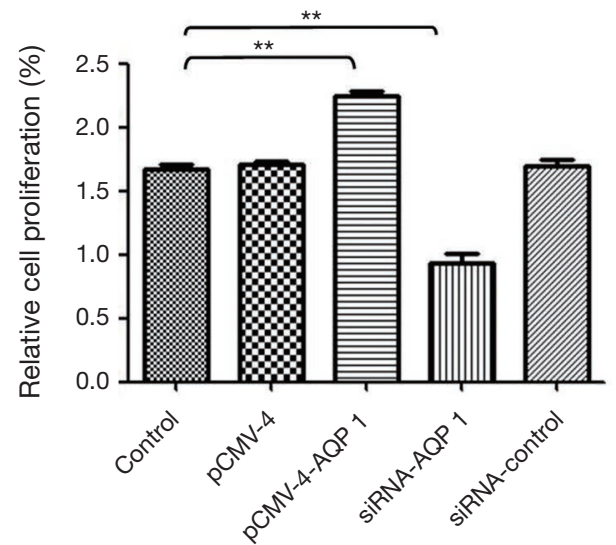

Figure $3 \mathrm{AQP}-1$ promoted growth of glioma cells. Glioma cells $(3 \times 103)$ were added into 96-well plates and cultured for $24 \mathrm{~h}$, then transfection with pCMV-4, pCMV-4-AQP-1, and the siRNA of AQP-1 or siRNA control. We conduct the MTS assay to assess the effect of overexpression or siRNA AQP-1 on proliferation of glioma cells. Compared with miR-NC cells, proliferation of U251 (A) and U87 (B) cells would be accentuated when they were transfected with pCMV-4-AQP-1, respectively. Moreover, the siRNA of AQP-1 attenuated these processes of glioma cells. The data are presented as means $\pm \mathrm{SD}$ from three independent experiments. ${ }^{* *}, \mathrm{P}<0.01$; $\mathrm{P}$ value was generated using Kruskal-Wallis ANOVA. AQP, aquaporin; ANOVA, analysis of variance.

ability of glioma cell lines U87 and U251 (Figure 4).

\section{AQP-1 accentuate migration of glioma Cells}

It demonstrated the migration ability was obviously promoted by AQP-1 compared to control in both cell lines U251 (Figure $5 A, B$ ) and U87 (Figure $5 B, C$ ) $(\mathrm{P}<0.001)$. Moreover, down-regulation of AQP-1 inhibited significantly ability of migration in both cell lines (Figure 5). The siRNA of AQP-1 obviously inhibited cell migration in U251 and U87 glioma cell lines, respectively. These results demonstrated AQP-1 could suppress tumor progression related to tumorigenicity in glioma.

\section{Discussion}

AQPs belong to channel and integral membrane proteins, and also MIP (major intrinsic protein) (20). Moreover, these proteins allow uncharged small molecules and water to passively permeate cell plasma membrane. According to the highly conserved structure, NPA (dual asparagineproline-alanine) boxes, AQPs could be recognized. NPA is critical for the formation pore for permeating of water. The classical sequences of AQPs all have six conserved transmembrane domains with likely repeated hydrophobic NPAs. Moreover, the family proteins have only minimal difference from others $(7,21)$. Furthermore, in motifs of NPA, only poor conserved sequence present in AQP-like sequences (22). The motifs of NPA in AQPs play the vital roles on premating cell membranes of water movement (20).

For evaluating the possible biotic outcomes induced by AQP-1, we over-expressed or down-expressed AQP-1 in $\mathrm{U} 87$ and $\mathrm{U} 251$, and then several experiments associated with cell functions were performed to evaluate tumorigenicity and progression of glioma. We conduct MTS assay to assess the effect of overexpression or siRNA AQP-1 on proliferation of glioma cells. Compared with miR-NC cells, cell growth of U87 and U251 were all accentuated by overexpression of AQP-1, respectively. Moreover, the siRNA of AQP-1 attenuated these processes of glioma cells (Figure 3). Furthermore, the colony formation assay revealed that AQP-1 exhibited significantly facilitated ability for colony formation compared with the control group in both cell lines U251and U87 $(\mathrm{P}<0.001)$. But, down-regulation of AQP-1 decreased significantly ability of colony formation in both cell lines. These results suggested that AQP-1 also suppressed the clonogenicity ability of glioma cell lines U87 and U251 (Figure 4). Our results demonstrated the migration ability was obviously promoted by AQP-1 compared to control in U251 and U87 $(\mathrm{P}<0.001)$. Moreover, down-regulation of AQP-1 inhibited significantly ability of migration in both cell lines (Figure 5). Our results indicated that the cell growth and migration of glioma cells could be inhibited by the treatment with down regulation of AQP-1 expression, but promoted by overexpression of AQP-1. Two metabolism models were 

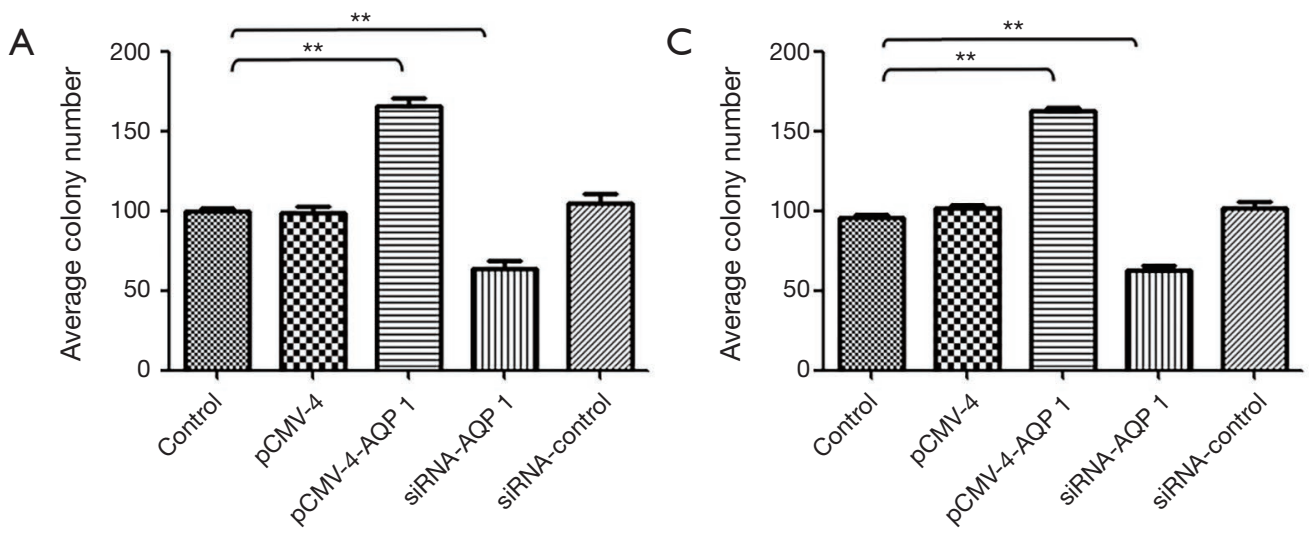

B

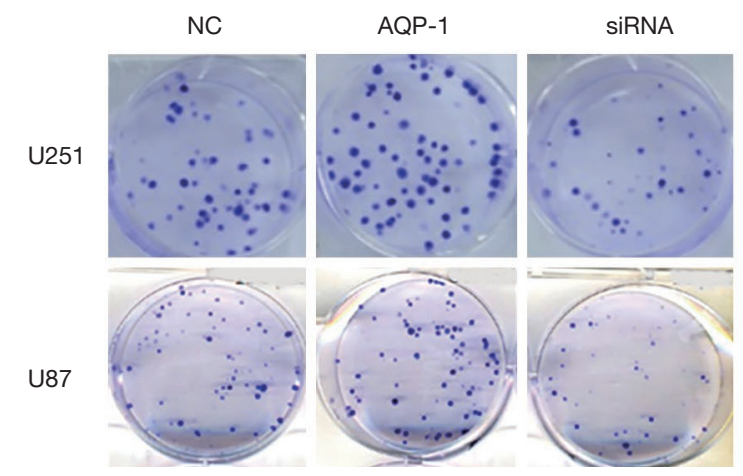

Figure 4 AQP-1 promoted the colony formation of glioma cells. Furthermore, the colony formation assay revealed that AQP-1 exhibited significantly facilitated ability for colony formation compared with the control group in both cell lines U251 (A,B) and U87 (B,C) $(\mathrm{P}<0.001)$. But, down-regulation of AQP-1 decreased significantly ability of colony formation in both cell lines (B, stained with crystal violet). The data are presented as means $\pm \mathrm{SD}$ from three independent experiments. **, $\mathrm{P}<0.01 ; \mathrm{P}$ value was generated using Kruskal-Wallis $\mathrm{ANOVA}$. AQP, aquaporin; ANOVA, analysis of variance.

established in human recently, the potential utility of them have been identified in clinic $(23,24)$. The common models in human could be used to investigate metabolism of tumor and activities of metabolism based on specific context generation $(25,26)$.

When the level of glucose in blood decreases caused by exercise or fasting, it activates gluconeogenesis in cells. Then the glucose homeostasis restored by glucose production. The gluconeogenesis is not active in all cells; cancer cells are known to have, instead, a very active glycolysis. There are four chief reactions involved in gluconeogenesis, which is accomplished by regulation of transcriptional, post-translational level induced by hormonal stimulus. G6Pase, F1, 6BPase, PEPCK, and PC catalyze these four reactions. The regulation of these enzymes in transcriptional level is vital to effectively induce gluconeogenesis (27). Then the key rate-limiting enzyme of these four reactions were investigated in this work, such as G6PC, GK, and GOT1.

Focusing on the mechanism of glioma cell growth and migration associated with AQP-1, the relationship between AQP-1 and glucose metabolism on tumorigenesis of glioma was investigated. Then, the role of AQP-1on gluconeogenesis was conducted to entirely elucidate and identify the key pathways of metabolism related to AQP1 in glioma, overexpression or knockdown of AQP-1 was induced in U87 and U251, and then the expression level of glycogenic gene or glucose production was examined.

The amounts of glucose production in glioma cells treat with overexpression of AQP-1 enhanced obviously. On the contrary, the amounts of glucose production reduced significantly induced by the siRNA of AQP-1. Our work demonstrated that $\mathrm{AQP}-1$ facilitated production of glucose in glioma cells, but reduced by siRNA of AQP-1. Moreover, over expression of AQP-1 caused high protein expression level of G6PC, GK and GOT1, but inhibited by siRNA of AQP-1. 
A

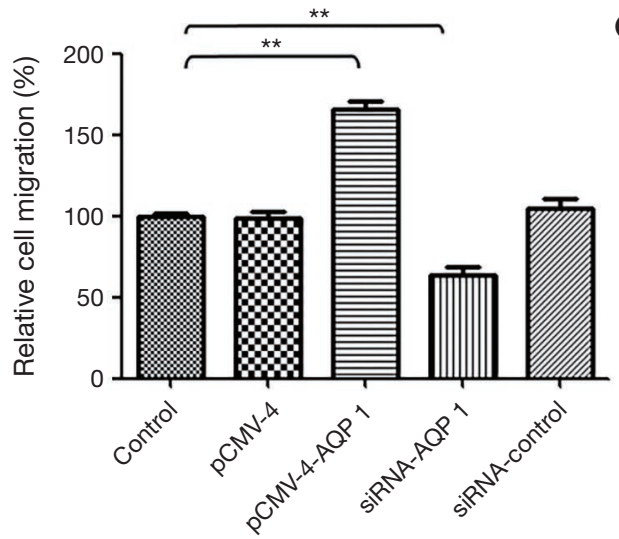

B

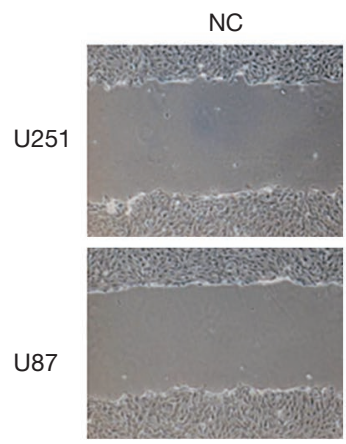

AQP-1

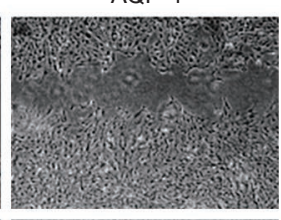

C

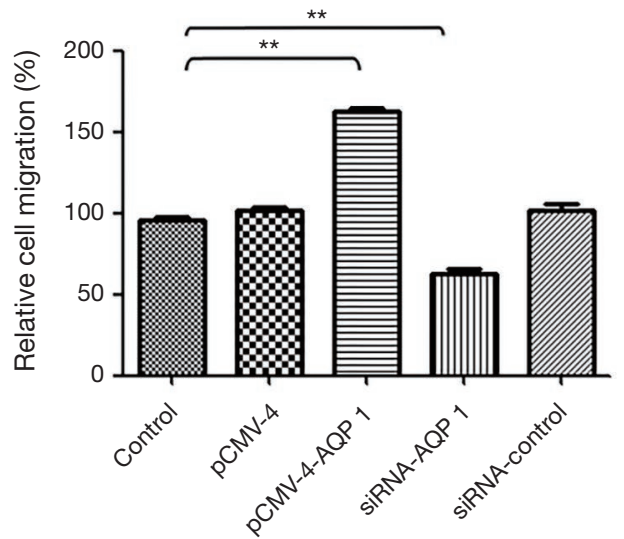

SiRNA

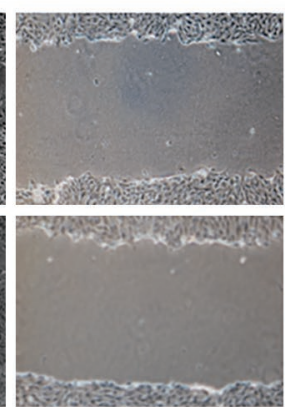

Figure 5 AQP-1 accentuate migration of glioma cells. It demonstrated the migration ability was obviously promoted by AQP-1 compared to control in both cell lines U251 (A,B) and U87 (B,C) (P<0.001). Moreover, down-regulation of AQP-1 inhibited significantly ability of migration in both cell lines. The siRNA of AQP-1 obviously inhibited cell migration in U251 and U87 glioma cell lines, respectively $(\mathrm{B}, 400 \times)$. The data are presented as means $\pm \mathrm{SD}$ from three independent experiments. ${ }^{* *}, \mathrm{P}<0.01$; $\mathrm{P}$ value was generated using KruskalWallis ANOVA. AQP, aquaporin; ANOVA, analysis of variance.

These data indicated that AQP-1 facilitated growth and migration of glioma cells by regulating glucose metabolism, which was related to down-expression of gluconeogenesis gene G6PC, GK and GOT1. Interference on AQP1 expression reduced these processes of glioma cells. Therefore, this work demonstrated that down regulation of AQP-1 can induce the inhibition of glioma cell growth and migration through the pathway related to gluconeogenesis gene G6PC, GK and GOT1, which may promote the glucose metabolism. AQP-1 is the potential novel target for glioma treatment.

\section{Acknowledgments}

Funding: This work was granted by the National Natural Science Foundation of China (No. 30600524 and 81341067), National Natural Science Foundation of
Guangdong Province, China (No. 2017A030313510), and Introduction of Talent Fund of Guangdong Second Provincial General Hospital (No. YY2016-006).

\section{Footnote}

Conflicts of Interest: All authors have completed the ICMJE uniform disclosure form (available at http://dx.doi. org/10.21037/tcr.2018.11.02). The authors have no conflicts of interest to declare.

Ethical Statement: The authors are accountable for all aspects of the work in ensuring that questions related to the accuracy or integrity of any part of the work are appropriately investigated and resolved. The study was conducted in accordance with the Declaration of Helsinki (as revised in 2013). Institutional ethical approval and 
informed consent were waived.

Open Access Statement: This is an Open Access article distributed in accordance with the Creative Commons Attribution-NonCommercial-NoDerivs 4.0 International License (CC BY-NC-ND 4.0), which permits the noncommercial replication and distribution of the article with the strict proviso that no changes or edits are made and the original work is properly cited (including links to both the formal publication through the relevant DOI and the license). See: https://creativecommons.org/licenses/by-nc-nd/4.0/.

\section{References}

1. Mamelak AN, Jacoby DB. Targeted delivery of antitumoral therapy to glioma and other malignancies with synthetic chlorotoxin (TM-601). Expert Opin Drug Deliv 2007;4:175-86.

2. Goodenberger ML, Jenkins RB. Genetics of adult glioma. Cancer Genet 2012;205:613-21.

3. Rachet B, Mitry E, Quinn MJ, et al. Survival from brain tumours in England and Wales up to 2001. Br J Cancer 2008;99:S98-101.

4. Ziu M, Kalkanis SN, Gilbert M, et al. The role of initial chemotherapy for the treatment of adults with diffuse low grade glioma: A systematic review and evidence-based clinical practice guideline. J Neurooncol 2015;125:585-607.

5. Chung S, Nakamura Y. MELK inhibitor, novel molecular targeted therapeutics for human cancer stem cells. Cell Cycle 2013;12:1655-56.

6. Engel A, Fujiyoshi Y, Agre P. The importance of aquaporin water channel protein structures. EMBO J 2000;19:800-6.

7. Verkman AS, Mitra AK. Structure and function of aquaporin water channels. Am J Physiol Renal Physiol 2000;278:F13-28.

8. Kuriyama H, Kawamoto S, Ishida N, et al. Molecular cloning and expression of a novel human aquaporin from adipose tissue with glycerol permeability. Biochem Biophys Res Commun 1997;241:53-8.

9. Hayashi Y, Edwards NA, Proescholdt MA, et al. Regulation and function of aquaporin-1 in glioma cells. Neoplasia 2007;9:777-87.

10. Hirschhaeuser F, Sattler UG, Mueller-Klieser W. Lactate: a metabolic key player in cancer. Cancer Res 2011;71:6921-5.

11. Van Hée VF, Pérez-Escuredo J, Cacace A, et al. Lactate does not activate NF- $\mathrm{KB}$ in oxidative tumor cells. Front Pharmacol 2015;6:228.

12. Schwab A, Stock C. Ion channels and transporters in tumour cell migration and invasion. Philos Trans R Soc
Lond B Biol Sci 2014;369:20130102.

13. Badaut J, Regli L. Distribution and possible roles of aquaporin 9 in the brain. Neuroscience 2004;129:971-81.

14. Qin YS, Bu DX, Cui YY, et al. Difference in glycerol levels between leukemia and normal bone marrow stem cells. Oncol Lett 2014;8:169-74.

15. Huber VJ, Tsujita $M$, Nakada T. Aquaporins in drug discovery and pharmacotherapy. Mol Aspects Med 2012;33:691-703.

16. Wang F, Feng XC, Li YM, et al. Aquaporins as potential drug targets. Acta Pharmacol Sin 2006;27:395-401.

17. Liu X, Chen J, Zhang J. AdipoR1-mediated miR3908 inhibits glioblastoma tumorigenicity through downregulation of STAT2 associated with the AMPK/ SIRT1 pathway. Oncol Rep 2017;37:3387-96.

18. Leconet W, Petit P, Peraldi-Roux S, et al. Nonviral Delivery of Small Interfering RNA Into Pancreasassociated Immune Cells Prevents Autoimmune Diabetes. Mol Ther 2012;20:2315-25.

19. Beitz E. Aquaporins from pathogenic protozoan parasites: structure, function and potential for chemotherapy. Biol Cell 2005;97:373-83.

20. Zeuthen T. How water molecules pass through aquaporins. Trends Biochem Sci 2001;26:77-9.

21. Zardoya R. Phylogeny and evolution of the major intrinsic protein family. Biol Cell 2005;97:397-414.

22. Ishibashi K. Aquaporin subfamily with unusual NPA boxes. Biochim Biophys Acta 2006;1758:989-93.

23. Duarte NC, Becker SA, Jamshidi N, et al. Global reconstruction of the human metabolic network based on genomic and bibliomic data. Proc Natl Acad Sci USA 2007;104:1777-82.

24. Ma H, Sorokin A, Mazein A, et al. The Edinburgh human metabolic network reconstruction and its functional analysis. Mol Syst Biol 2007;3:135.

25. Jerby L, Shlomi T, Ruppin E. Computational reconstruction of tissuespecific metabolic models: application to human liver metabolism. Mol Syst Biol 2010;6:401.

26. Frezza C, Zheng L, Folger O, et al. Haemoxygenase is synthetically lethal with the tumour suppressor fumaratehydratase. Nature 2011;477:225-8.

27. Jitrapakdee $S$. Transcription factors and coactivators controlling nutrient and hormonal regulation of hepatic gluconeogenesis. Int J Biochem Cell Biol $2012 ; 44: 33-45$.

Cite this article as: Liu X, Chen J, Zhang J. To inhibit growth and migration of glioma cells through down regulation glucose metabolism related to aquaporin (AQP)-1. Transl Cancer Res 2018;7(6):1413-1420. doi: 10.21037/tcr.2018.11.02 